\title{
Development and Usability Testing of Simulated Wind in a Racing Video Game
}

\author{
Miguel A. Garcia-Ruiz \\ Dept. of Mathematics and Computer Science \\ Algoma University \\ Sault Ste. Marie, Canada \\ miguel.garcia@algomau.ca
}

\author{
Pedro C. Santana-Mancilla \\ School of Telematics \\ University of Colima \\ Colima, Mexico \\ psantana@ucol.mx
}

\begin{abstract}
We present a development and a user study on simulated wind in a racing video game. Our research question asked whether low-cost technology could be effective for making simulated wind, and how it could affect the racing game's usability. Two computer fans were operated by a microcontroller that was connected to a $\mathrm{PC}$ running a racing video game. The fans' speed changed according to the car acceleration in the game. We conducted a Concurrent Think Aloud Protocol usability test, where 16 participants played the video game. At the end of the usability test, participants filled out a System Usability Scale (SUS) questionnaire. Results indicate that all the participants felt the simulated wind usable for the game. Results also indicated that the game usability was not affected by the simulated wind, and the software/hardware configuration can be a cost-effective solution for simulating wind. Further work include experimenting with more powerful fans.
\end{abstract}

Keywords-Video game; wind simulation; racing game; usability testing; multimodal interfaces.

\section{INTRODUCTION}

Multimodal video game interfaces have multiple input and output communication channels using various human senses. They allow users to interact more naturally with video games $[1,2]$. In racing video games, players typically use their visual, auditory and touch (haptic) sensory channels by looking at the screen, listening to the game sound effects, steering a gaming wheel, and stepping on its pedals. Simulated wind in video games can be part of the touch sensory channel, where players feel the wind hitting the players' skin and face. This paper presents a development and testing of simulated wind in a racing video game. Our research question asked whether low- cost technology could be effective for making simulated wind in a desktop PC, and how it could affect the usability of a racing game. Usability is the ease of use of a human-computer interface. It generally measures the efficiency, effectiveness and satisfaction of use of digital devices [3], including video games.

\section{LITERATURE REVIEW}

There are a number of projects regarding wind simulation in computer simulations and video games. One of the first applications of simulated wind in interactive interfaces was the Sensorama. It was an arcade-like personal simulator that displayed an interactive movie, where the user perceived odors, felt vibrations, and felt simulated wind according to the events shown in the projected movie, for example, when the interface displayed a scene about riding a motorcycle [4].

Deligiannidis and Jacob [5] developed and tested a "virtual scooter" where users could steer a scooter-like prototype made of wood and sensors in a 3D virtual environment, as if they were riding a real scooter. It had simulated wind blowing at the user using fans. The wind intensity was adjusted according to the velocity of the virtual scooter. A usability test found that participants improved their navigation time using the virtual scooter with the simulated wind. Also, most of the participants liked the use of the fans for feeling the sense of speed better.

\section{WIND SIMULATION DEVELOPMENT}

In order to answer our research question, we used two 12$\mathrm{cm}$. PC fans operated by an Arduino Uno microcontroller connected to the $\mathrm{PC}$ running a racing video game. A program written in Python controlled the fans' speed according to the car acceleration in the game. It is interesting to note that the fans, the microcontroller and the program could be used in any racing game having sound effects, such as the engine sound made when the player is accelerating. The program analyzed the engine sound frequency and adapted the fans speed accordingly. Players used Vdrift [6], a cross-platform, opensource racing video game running on Ubuntu Linux. Players also used a Logitech G27 racing wheel and pedals [7]. Figure 1 shows the video game set up.

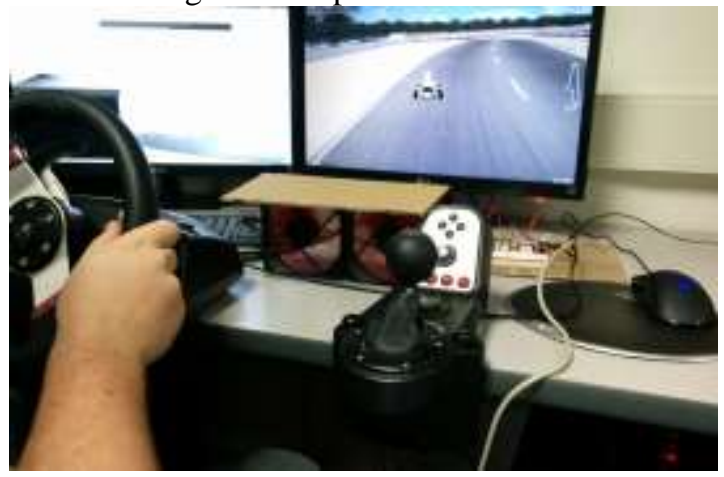

Fig. 1. Fans and video game set up. 
The fans were placed approx. 1 meter apart from the face of the player. We sticked a piece of carton on top of the fans because we did not want the players to see them rotating and just perceive the simulated wind, in order to avoid visual feedback on their rotation. Figure 2 shows the wind simulation and video game configuration.

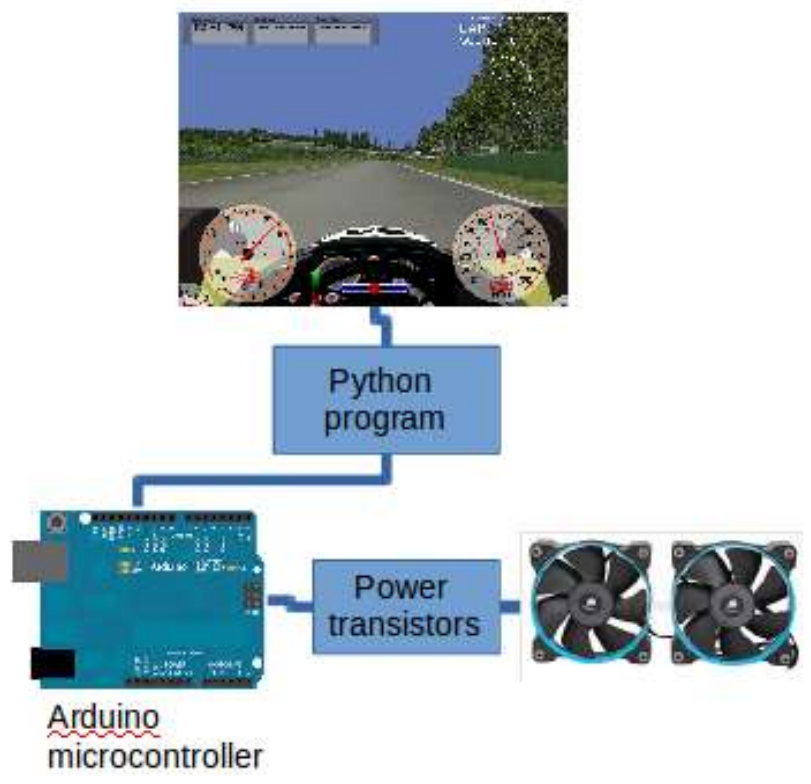

Fig. 2. Video game and wind simulation configuration.

\section{UsABILITY TEST}

We tested the usability of the fans and the racing game using the Concurrent Think Aloud Protocol (CTAP) [8] method. The CTAP is a practical usability method where participants test out a computer system, including its user interface. In it, participants are asked to say out loud their thoughts and actions about the interface while they are interacting with it. The CTAP is useful for testing gaming interfaces since it provides qualitative data on the tactile, auditory and visual aspects of the game, as well as the type of tasks participants require to do in the game [10].

Participants in the CTAP were 16 computer science students with an age average of 20 years, all males. Half of them have played racing games before. At the beginning of the test, each participant had a few minutes' training session. The main task in the game was to drive a cart for ten minutes using the racing wheel, accelerating and decelerating every minute sequentially to perceive changes in the wind speed. Experimenters were taking notes on the participants' verbal comments while the participants played the game, as per the CTAP guidelines. At the end of the usability test, participants filled out the reliable System Usability Scale (SUS) questionnaire [9]. It is an industry-standard questionnaire that measures the usability of digital products, including video games [10]. The SUS questionnaire is composed of 10 items each with five response options in the form of Likert scales; from Strongly Agree to Strongly Disagree. This questionnaire is scored and provides a usability percentile ranking, where $0=$ very poor usability and $100=$ excellent usability.

\section{RESULTS AND CONCLUSIONS}

Results indicate that most of the participants (90\%) felt that the wind simulation was quite usable a non-obtrusive. Results also show that the wind simulation running along with the video game was pleasant to perceive by all the participants. The average score from the SUS questionnaire was 85. According to participants' comments made during the CTAP, further developments in our wind simulation should include the use of more powerful fans in the video game, with the objective of generating more wind and increasing realism. Further work will also experiment simulation of wind direction coming from a specific side if the player turns the cart in the game to the left or right, or if the incoming wind change its direction.

Results from this study positively answered our research question, since low-cost technology such as the microcontroller and the fans used in it can be effective for making simulated wind in a desktop PC, without affecting the overall usability of a racing game. In addition, open-source software, such as the racing simulator and the Python interpreter used in our study, can be an affordable and effective solution for people or educational/research institutions who wish to develop and apply a multimodal video game/car simulator with wind simulation.

\section{REFERENCES}

[1] S. Oviatt, "Multimodal interfaces", Handbook of Human-Computer Interaction, 3rd. ed., edited by J. Jacko, New Jersey: Lawrence Erlbaum, 2012.

[2] E. Tse, S. Greenberg, C. Shen, \& C. Forlines, C., "Multimodal multiplayer tabletop gaming," Computers in Entertainment (CIE), 2007, $5(2), 12$.

[3] J. Nielsen, "Usability 101: Introduction to Usability", Nielsen Norman Group, 2012. Avaliable from: http://www.nngroup.com/articles/usability-101-introduction-to-usability/

[4] S. Fisher, "Virtual Environments, Personal Simulation, \& Telepresence" in Virtual Reality: Theory, Practice and Promise, S. Helsel and J.Roth, ed., Meckler Publishing, 1991.

[5] L. Deligiannidis and R.J. Jacob, "The vr scooter: Wind and tactile feedback improve user performance", In IEEE Symposium on 3D User Interfaces, 2006, pp. 143-150.

[6] VDrift. (2012) "About VDrift". [Online]. Available: http://vdrift.net/

[7] Logitech. (2010) "G27 Racing Wheel". [Online]. Available: http://gaming.logitech.com/en-us/product/g27-racing-wheel

[8] M. Van Den Haak, M. De Jong and P. Jan Schellens, P. (2003), "Retrospective vs. concurrent think-aloud protocols: testing the usability of an online library catalogue" Behaviour \& information technology, 2003, 22(5), 339-351.

[9] J. Brooke, "SUS-A quick and dirty usability scale", Usability evaluation.

[10] J.M., Garcia-Diaz, J. M., Garcia-Ruiz, M. A., Aquino-Santos, R., and A. Edwards-Block, "Evaluation of a Driving Simulator with a visual and Auditory Interface", Human Computer Interaction. Lecture Notes in Computer Science, Springer, Volume 8278, pp 131-139, 2013. 